\title{
4. Eye Movements and an Object-Based Model of Heading Perception
}

\author{
Ranxiao Frances Wang ${ }^{1}$ and James E. Cutting ${ }^{2}$ \\ ${ }^{1}$ Department of Psychology \\ University of Illinois, Champaign, IL, USA \\ ${ }^{2}$ Department of Psychology \\ Cornell University, Ithaca, NY, USA
}

\section{INTRODUCTION}

The movement of an observer through an environment of stationary objects creates a complex set of motions called optical flow. Accurately registering these motions with our mobile eyes and acting upon them underlies the everyday commerce and safety of all animals, particularly human beings whose technological means of conveyance have accelerated both speeds and risks. Optical flow is rich and can be parsed in many ways (e.g., Cutting, 1986; Gibson, 1966; Koenderink \& van Doorn, 1975; Regan \& Beverley, 1982; see Lappe et al., 1999; and Warren, 1998, for reviews). There are many tasks that depend, at least in part, on optical flow - maintaining balance, detecting potential collisions, and monitoring the course of navigation. In this chapter, we discuss a model of heading perception that underlies navigation. It is based on relative motions of object pairs and on eye movements with which observers seek them out prior to heading judgments.

\section{MODELS OF HEADING JUDGMENTS}

Most approaches to understanding heading judgments generally have two characteristics. First, they focus directly on obtaining absolute heading information, the precise location of one's instantaneous aim point. Second, rather than focusing on (a) objects, (b) relative motion, and (c) relative depth, these approaches focus on either (b) motion alone, (b\&c) motion and depth, or 
(a\&c) objects and depth. And finally, one approach considers all of this largely irrelevant. Consider each in turn.

\subsection{Motion Alone}

There are at least two computational schemes that have been posited. Both deal with sectors of the visual field - that is, regions with respect to the fovea. The first is differential motion, proposed by Rieger and Lawton (1985) and elaborated by Hildreth (1992). It squares the length of each motion vector and then adds all vectors within a sector. Second, spatial pooling (Warren \& Saunders, 1995) simply adds each vector within a sector. Across sectors heading is determined by comparing the directions and extents of summed vectors. Our implementations of these schemes (Cutting et al., 1999) have shown that the spatial pooling fares better than differential motion. However, we also found that the presence and absence of the invariant cues (as discussed below) predicted heading results of human observers much better than either pooling scheme.

\subsection{Motion and Depth}

Perrone \& Stone (1994, 1998; see Grossberg et al., 1999) presented a model based on their synthesis of anatomy, physiology, and data concerning visual area MST. This scheme is a bit like the models just considered except that motions are also pooled at different depths. This approach can account for virtually all data, but it is also not easily falsifiable. However, since all regions in space and depth are considered essentially equal, there is no role for attention, and we know that attention selects out particular objects of interest. Motter (1993), among others, has shown that responses of single cells in many visual areas are modulated by attention paid to particular objects in motion. These suppress activity that is otherwise present in the classical receptive field. Our notion is that human observers pay attention to particular objects and that it is their relative motion, not the global array of motion, that matters for finding one's way.

\subsection{Objects and Depth}

Another approach does not directly consider motion or its patterns. Frey and Owen (1999) proposed a measure of information in a heading display, called the separation ratio. This ratio, $\sigma$, can be expressed as: 


$$
\sigma=1-n / f
$$

where $n$ is the distance from the observer to the nearest object at the end of the sequence and $f$ that to the farthest. Its value always falls between 0 and 1 . Frey and Owen suggested that this ratio should predict heading performance, the greater the ratio the more accurate observers' responses. Indeed, typically in our displays the value of this ratio is reliably correlated with mean heading judgments. However, in two data sets (Cutting et al., 2000; Cutting \& Wang, 2000), a stepwise multiple regression was performed with two predictors of absolute heading performance - the separation ratio, and the presence (coded as 1) or absence (coded as 0) of invariants on any given trial. We found that the invariant code accounted for $35 \%$ of the variance in the data, and the separation ratio only an additional $4 \%$. Thus, the separation ratio is correlated with the invariant information in these displays, but by itself does not substantially contribute to the responses. Differential motion and spatial pooling also accounted for no appreciable variance.

\subsection{Visual Direction}

Rushton and co-workers (1998) and Llewellyn (1971) proposed that optical flow is not paramount for determining one's heading. All one need to do is rely on visual direction - for example, pointing one's head and body towards a landmark one wishes to attain and simply walking there, allowing the muscles involved in the orientation of one's eye and head to guide one's movements. Indeed, manuals of motorcycle riding (Motorcycle Safety Foundation, 1992) and equitation (Morris, 1990) support this idea and it undoubtedly plays a role in wayfinding. However, Warren and co-workers (2001) showed that both sources are used, with optical flow information dominating when observer-relative velocities increase. Thus, visual direction seems to be a framework within which optical flow makes its major contribution.

\section{OUR APPROACH}

In analyzing the available information for the perception of one's heading, we have found it useful to start with the relative motions of pairs of objects. These can yield nominal information (left or right of a landmark) about heading. When coupled, multiple pairs can yield near-absolute information - a heading specification that fall between two landmarks in the 
visual field. In this section, we discuss three object-based sources of information for heading judgment.

\subsection{Object-Based Information}

Several classes of pairwise motions occur in the forward visual field: Objects converge or diverge. If the latter, they decelerate apart or accelerate apart. All convergence in the forward field is acceleratory. These three classes of relative motions, and their consequences for heading location, are suggested in Figure 1a. They apply to both straight and curved paths (Wang \& Cutting, 1999b). If a moving observer knows the relative motion and the ordinal depth of two objects - which is nearer and which farther away then several strong statements can be made concerning his or her nominal heading.

First, pairs that converge specify an invariant relation among themselves, a moving observer, and his or her heading. Heading is always to the outside of the nearer member of the pair, regardless of observer velocity or where in the field of view the pair might appear, as shown in Figure 1b. There are no exceptions. As discussed later, convergence is sufficiently potent that Wang and Cutting (1999a) implemented a machine algorithm for heading detection that works without assumptions of depth order; that knows only that heading can never lay between converging stationary objects.

Second, for pairs that decelerate apart, heading is also to the outside of the near member of the pair, but only when both objects are within $45^{\circ}$ of the heading. This constraint is not too limiting since the data of Wagner, Baird, and Barbaresi (1981) show that pedestrians look within this limit about $90 \%$ of the time (see Cutting et al, 1999).

Third, without other information, pairs that accelerate apart offer no firm statement about heading. Probabilistically, one can state that heading is most often to the outside of the farther member of the pair - $69 \%$ of the time as calculated by Wang and Cutting (1999b) and shown in Figure 1c. Thus, accelerating divergence is a heuristic, not an invariant (Cutting \& Wang, 2000; see also Gilden \& Proffitt, 1989).

Fourth, there are pairs that have crossed over one another in the field of view. If one can remember the crossover of a pair of objects, heading will always be outside its farther member. This too is an invariant. It follows from the fact that converging items will, if the observer continues long enough on a straight path, always meet in the field of view, the nearer occlude the farther, and the two accelerate apart. Thus, what separates these third and fourth sources is, in part, observer memory. However, whereas all crossover pairs accelerate apart, all pairs accelerating apart need not have crossed over. Any 


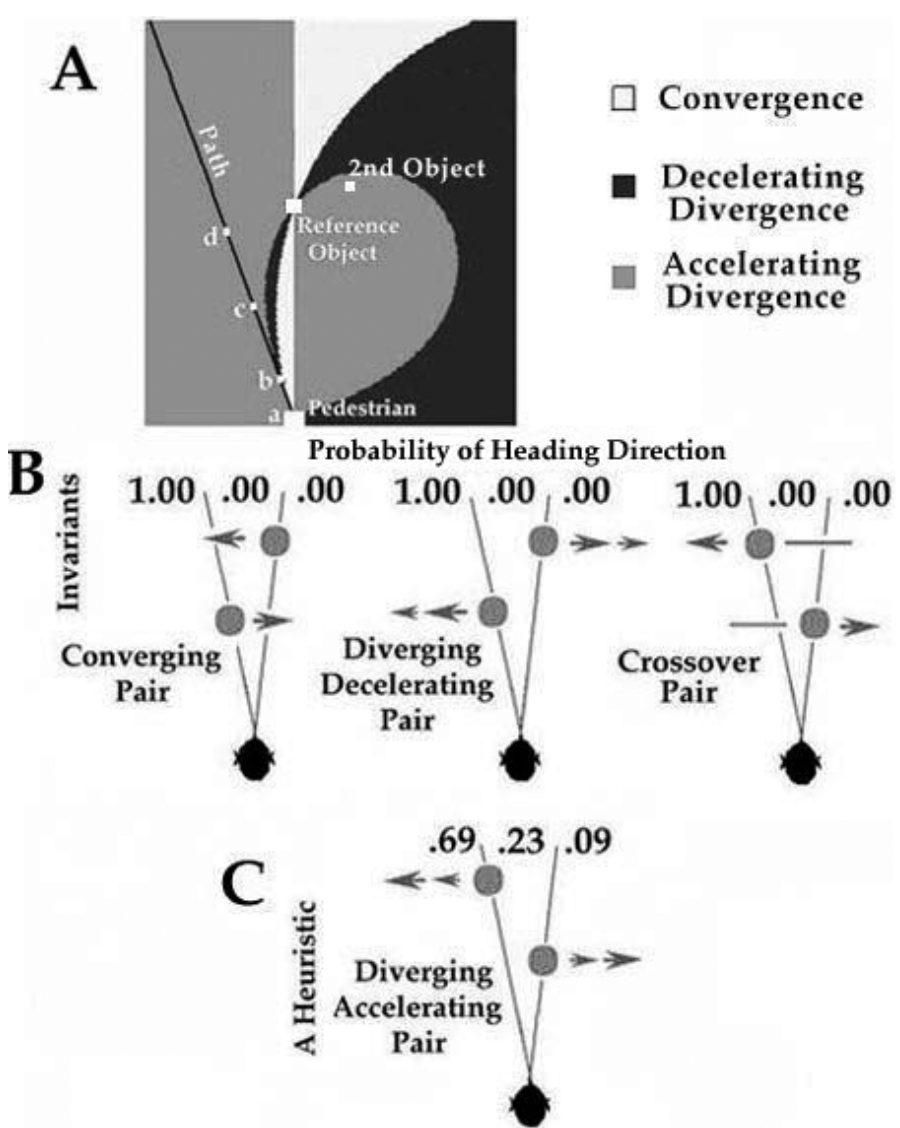

Figure 1. Panel A is a plan view of the layout around a pedestrian, and relative motions around an object $20^{\circ}$ off path to the right. All other objects converge, diverge and decelerate, or diverge and accelerate from it. Panels B show invariant relations for pairs specifying heading direction. For pairs converging or decelerating apart, heading is always outside the nearer object; for crossover pairs it is always outside the farther object. Panel C shows that heading direction is probabilistic for pairs that decelerate apart, but most often to the outside of the farther object. A fixed sequence can occur among these four relative motions, suggested in Panel A: At Point (a) the reference and 2nd objects accelerate apart, at Point (b) they decelerate apart, at Point (c) they converge, and at Point (d) they cross over.

change in direction of the moving observer will change the orientation of the patterns as seen in Figure 1a, and create a plethora of new pairs accelerating apart that never crossed over from the point of view of the observer. 


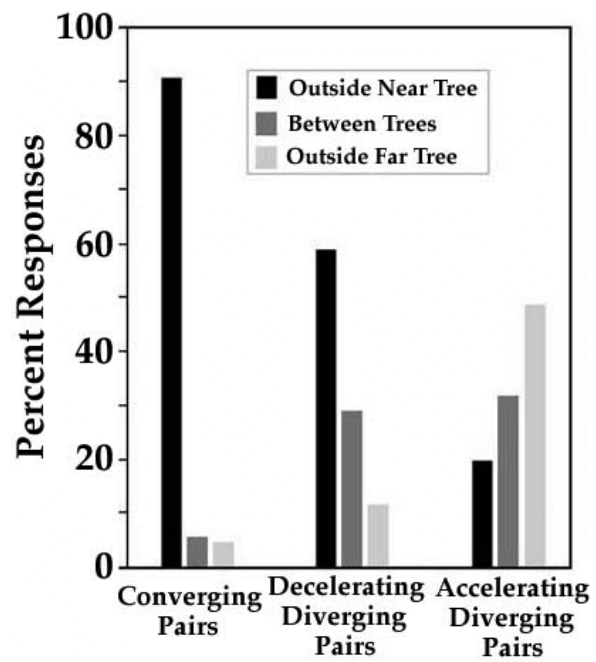

Figure 2. Heading responses for three types of stimuli in environments with only two trees. Responses are divided into three categories - those outside the near and far trees and those between. The information in Figure 1b would predict heading judgments should be most often placed outside the near tree for pairs that converge and decelerate apart, and outside the far tree when they accelerate apart. Results from Cutting \& Wang (2000) showed three reliably distinct patterns, and that these predictions were upheld. Convergence is the more salient (and powerful) invariant, and the heuristic of accelerating divergence yields the least diverse pattern of responses. There were no crossover trials in this study. See also Best et al (2002) for a replication.

\subsection{Invariants and Heuristic Compared}

How effective are our posited sources of information for human observers? To eliminate other factors Cutting and Wang (2000) used minimal stimuli, those with two trees and also no crossovers. They used a pursuit fixation technique (Cutting, 1986; Kim et al., 1996; Royden et al., 1992; Warren \& Hannon, 1988), with the dolly and pan of a camera simulating observer translation and eye/head rotation. Maximum rotations were less than $1 \%$, and thus eye muscle feedback is not necessary (Royden, et al, 1992). From Figure 1b, observers ought to place their heading to the outside of the near tree of invariant pairs that converge or decelerate apart. Indeed, as shown in Figure 2, observers followed this placement $91 \%$ of the time for convergent pairs and 58\% for pairs decelerating apart. These results show, as also found by Wang and Cutting (1999b) and Cutting et al (2000), that convergence is the more salient and powerful invariant. 


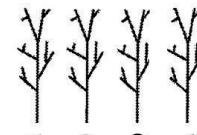

$\begin{array}{llll}1 & 2 & 3 & 4\end{array}$
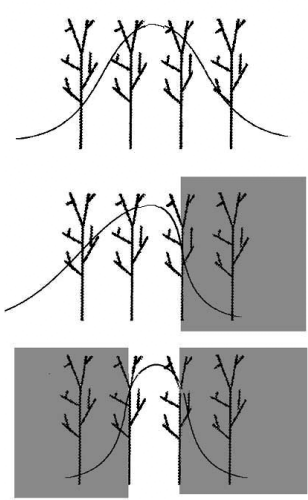

Ordinal Array

of Trees

No Invariants,
No Exclusions

One Invariant, One Exlcusion Boundary

Two Invariants, Two Boundaries of Opposite Sign

Figure 3. Schematic versions of four-tree stimulus arrays, diagrams of response distributions, and the response constraints in the three cases (in gray). For stimuli with no invariants, responses were distributed normally with a standard deviation (sd) of $3.6^{\circ}$. With one invariant the sd was $2.6^{\circ}$ and the distribution highly skewed. An example is shown where an invariant pair, Trees 3 and 4, constrain responses to the left of Tree 3. With two invariants of opposing sign, responses can be constrained to a region between two trees (here Trees 2 and 3). Here again they were normally distributed, but with an sd of only $1.4^{\circ}$. Mean errors also decreased across stimulus types - from 4.1 to 2.2 to $0.8^{\circ}$. Required heading accuracy in this situation is $3.5^{\circ}$ (Cutting et al, 1992).

Figure 1c suggests that for heuristic pairs, on the other hand, observers should place their heading to the outside of the far tree. And indeed they did - $47 \%$ of the time as shown in Figure 2 (see also Best et al., 2002). After considering these information sources, regression analyses showed no significant contributions of other variables, such as the angular separation of the two trees, or their relative distances from each other or the observer. The role of the heuristic in heading judgments with multiple objects is still not clear. What is clear is that the heuristic information cannot be combined with invariant information in the same manner the invariant information is combined. Thus, if accelerating divergence plays a role in heading judgment with multiple objects, some form of probability measure needs to be used. One such mechanism is illustrated in the next section on the simulations. 


\subsection{Invariants and Absolute Heading}

Accurate judgments of absolute heading can be achieved with invariants of opposing sign. Wang and Cutting (1999b) investigated such situations and Figure 3 shows a schematic rendering of how multiple invariants operate. In arrays without invariants responses were normally distributed; in arrays with one invariant responses were less varied and skewed away from the boundary specified by the invariant pair; and in arrays with two opposing invariants, with a permissible response region between two trees, responses were normally distributed but with a standard deviation less than half that of arrays without invariant pairs. Perhaps most importantly, absolute heading accuracy was within $0.8^{\circ}$, a value unsurpassed in the literature.

Wang and Cutting (1999a) developed an algorithm based on convergence alone. To compute the horizontal heading direction, the algorithm first divides the visual field into $n$ regions of equal size. At the beginning, it assumes that heading can be at any of these regions, with equal probability $(1 / n)$. Then it computes the relative motion of objects in a pair of the regions to determine whether there is a converging pair. If there is a converging pair, then heading is more likely to be toward the regions outside the pair. Thus, the probability rating of these regions increases, while that of the other regions decreases. If there is no converging pair, then the opposite happens (see Figure 4a). This process continues until the algorithm goes through every pair. The final heading judgment is determined by the region with the highest probability rating (see Figure 4c).

Using this algorithm, Wang and Cutting (1999a) examined the effects of the number of objects in the environment, the depth variations, and the rotation rate on the accuracy of heading judgments. In the simulation, an observer moved toward a dot cloud with different densities of the dots, different depth separation between the nearest dot and the farthest one, and different rotation rates. Then the retinal motion of each dot was calculated. Based on the retinal motion, the algorithm then computed the heading based on the relative velocity of pairs of the dots, by adjusting the probability rating for each region according to the rule of convergence. Finally, the angular error between the computed heading direction and the actual heading direction was calculated.

The simulation results suggest that the algorithm provides both accurate heading judgments and a pattern of errors consistent with that of human observers. The algorithm achieves heading judgment accuracy of 0.1 degree even with the rotation of the eyes, outperforming many field-based models in metric accuracy, even though the model itself is not metric. Moreover, accuracy improves as the number of dots increases, consistent with the 

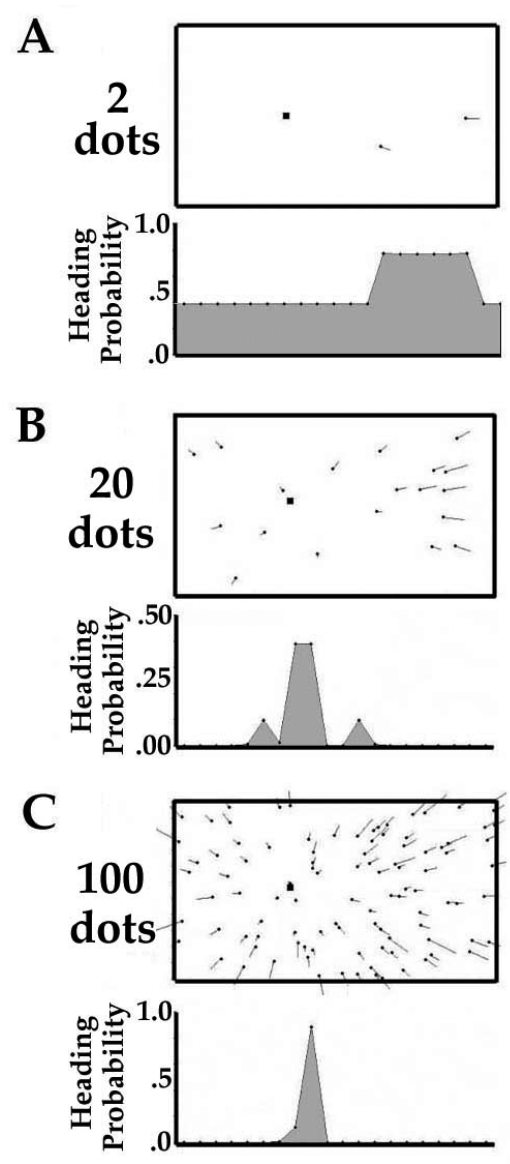

Figure 4. Simulation results of the algorithm based on convergence without depth information. Panel A shows a sample screen and results for two dots; Panel B for 20 dots, and Panel C for 100 dots. The small square shows the actual heading. The algorithm divides the horizontal visual field into 20 regions, and computes the probability of heading for each region. The judged heading is determined by the region with the highest probability. Notice that accuracy increases greatly with the number of dots (objects) in the display using one aspect of a single invariant, i.e., that one's heading can never lay between converging objects.

observation that human heading judgments are more accurate in richer environments (Li \& Warren, 2000; Warren \& Hannon, 1988). Accuracy also increases as a function of the depth variation. When the dots were all on a single frontal-parallel plane, heading error was rather large. As the depth separation among the dots increased, heading judgments became more accurate. 


\subsection{Invariants and Detecting a Moving Object}

Objects in the environment often move. Such objects could pose a threat to travel, or alternatively they might be food or an object to catch. In either case it would behoove an observer to be able to segregate motion (the independent translation of objects) from movement (object displacements due to observer translation), a distinction first made by Gibson (1954). Consider three ways. First, familiarity with objects will go some way towards specifying what is in motion and what is not. Cars, people, and animals can translate to new positions; trees, buildings, and rocks normally cannot. But, of course, at any given moment cars and other objects can be either translating or be stationary. Thus, familiarity cannot be the sole means by which motion among movement is detected.

Second, binocular disparities might aid the segregation of motion from movement (Kellman \& Kaiser, 1995). However, these may not be useful in situations where object motion is very slight, or when it is further away than about $30 \mathrm{~m}$ (Cutting \& Vishton, 1995).

Third, retinal velocity might predict their difference (Brenner, 1991; Wertheim, 1995). Wagner et al (1981) found that pedestrians look at stationary objects about $60 \%$ of the time, and moving objects $40 \%$. If we fixate a stationary object near our path, any object in motion is likely to be the fastest moving object in the field of view, or at least near the fovea, unless its velocity is quite minimal. If one fixates an object in motion the patterns of movement of stationary objects are complex, but such movements are critical for detections of collisions and bypasses (Cutting, Vishton, \& Braren, 1995).

Cutting and Readinger (2002) removed familiarity cues, binocular disparities, and insofar as possible, differences in retinal motion as variables that could aid in the detection of motion during movement. They presented viewers an array of identical poles, one of which (albeit perhaps somewhat mysteriously) was mobile, but only to a very modest degree. A sample stimulus arrangement is shown and explained in Figure 5. Observers indicated which pole was in motion during their simulated translation, and they could see each trial as often as they liked.

Detection of the mobile pole was strongly influenced by the field of invariants on a given trial. That is, if all invariants were coherent - they all specified that heading was in the same direction or they all converged uniformly on a central region - then judgments were less accurate $(47 \%$ correct where chance was 10\%) and more difficult (observers chose to see each such trial more often). When the invariants of the stimuli array were

incoherent, as shown in Figure 5, detection accuracy was greater (60\%) and less difficult. Moreover, if the trial was incoherent and the mobile pole 


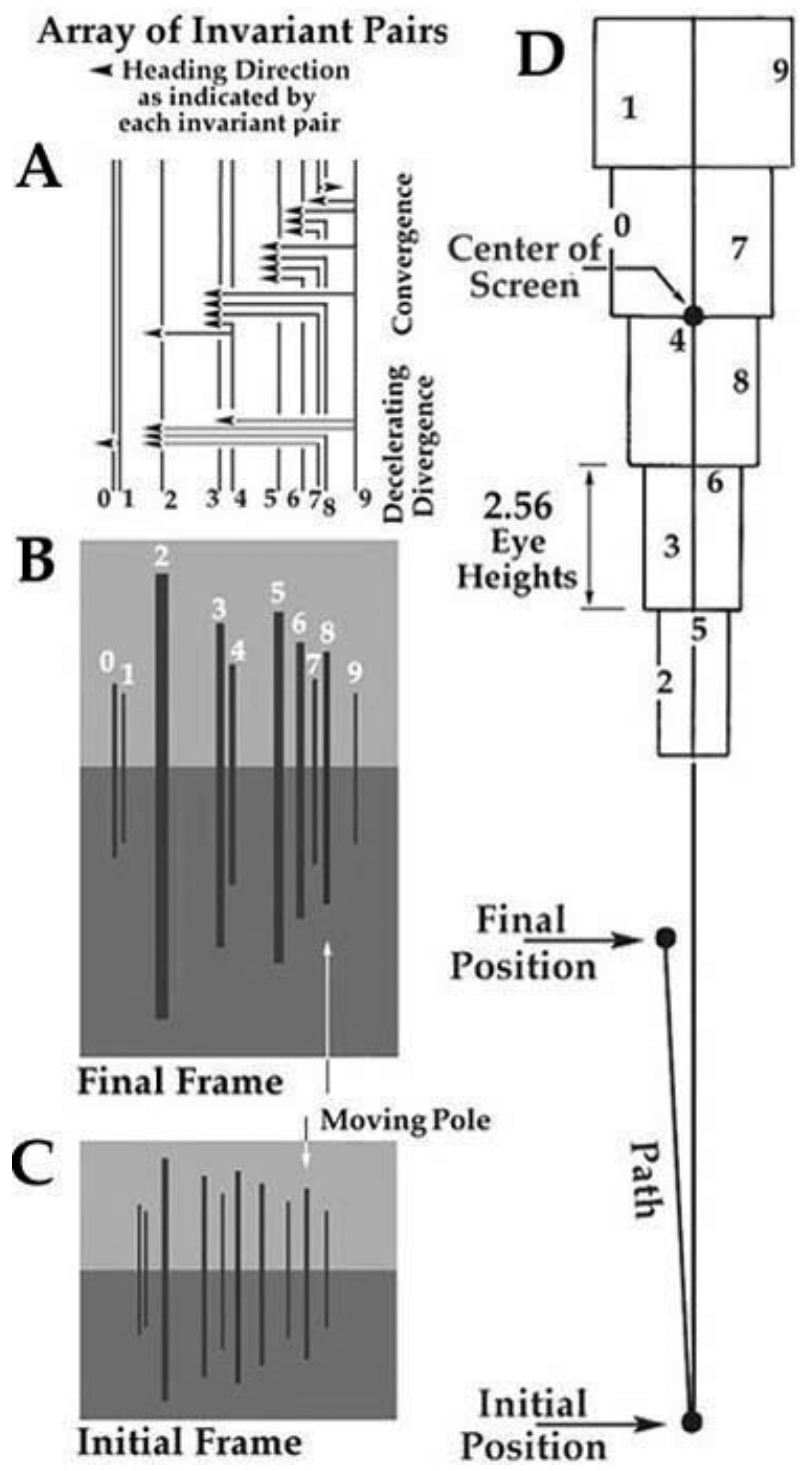

Figure 5. An example trial and its structure. Panel D shows a plan, scaled view of its layout, the locations of the observer, and the regions within which poles could be randomly placed. Panels $\mathrm{C}$ and $\mathrm{B}$ show the beginning and ending frames, respectively, of the four second sequence simulating observer movement along a path $3^{\circ}$ to the left of the poles and with a pan to the right to keep the center of the region of poles in view. The moving pole on this particular trial is Pole 8. Pole numbers and positions correspond in all panels. Panel A shows the 19 pairs of 
misidentified, two thirds of all confusion errors involved a neighboring pole that was involved in the invariant incoherent with the rest of the field. These results cannot be explained by image velocities of moving poles as opposed to the stationary ones or the relative depth of the moving pole within the display.

\section{EYE MOVEMENTS AND HEADING JUDGMENTS}

Animals and humans move their heads and eyes to reduce blur, particularly when they move (Land, 1999). Eye and head movements assure optimal resolution of objects of interest around them. But what do we look at, and why? Unfortunately, the data from free-ranging pedestrians is scant. Eye movement data from motorists have been discussed at length (e.g., Chapman \& Underwood, 1998; Land, 1992; Land \& Lee, 1994; see also Readinger et al., 2002). Although valuable, these data show that motorists often look at things that are not pertinent to a pedestrian (e.g. such as the inner tangent of a roadway during a turn). In addition Calvert (1954) observed that, as motorists increase their speed, they scan a smaller sector of the visual field, narrowing in on a region near their aim point. This is due to the fact that, with speed, accurate pursuit fixation off to the side becomes increasingly difficult. Motorists thus face a situation foreign to our evolutionary ecology. With an eye height less than a pedestrian and a translational velocity often an order of magnitude greater, they commonly experience a rapidity of optical flow never seen on foot, and never experienced by human beings before the mid- $19^{\text {th }}$ century (Schivelbusch, 1986). Motorists' fixations, eye movements, and skills are a marvel of human adaptability, but they cannot be mapped in a straightforward manner onto situations and constraints in which we evolved.

Important eye movement data also exist for pedestrians whose footfall is unsure (e.g., Hollands et al., 1995; Patla \& Vickers, 1997), suggesting ways in which we guarantee our safe progress by looking at a surface of support. However, in most situations we are relatively assured of our locomotion and not endangered of imbalance. In such cases, we do not look down very often; only $25 \%$ of the time do we look within 4 eye heights (about $6.4 \mathrm{~m}$ ) of our feet (Wagner et al, 1981). Thus, we have been interested in the looking behavior of pedestrians who would be secure in their gait and how they pick

Figure 5 cont'd: poles involved in heading invariants, 14 converging and 5 decelerating apart. Each arrow points in the specified heading direction, its stem connects the two poles involved in a particular invariant relation, and the base of the arrowhead delimits the edge of the response region allowed by the particular invariant pair. Eighteen of these invariants specify that heading be to the left; one pair involving Pole 8 yields a heading result incoherent with the rest, specifying that heading be to the right. This discrepancy would contribute to the detection of Pole 8 as a mobile pole, and also the possible confusion of Pole 7 with it. Were all invariant pairs consistent in their specified heading direction the trial would be called coherent. 
their paths through environments containing interesting things.

Recently, investigators have begun to consider the relation between eye movements and heading judgments. Some research has focused on whether, and how fast, observers can move their eye towards their heading (Hooge et al., 1999). Others have focused on whether eye movements are needed for heading judgments in unstructured environments with brief-display times (Grigo \& Lappe, 1999). Neither of these research foci is of direct interest here. Much research has demonstrated that people can find their heading rapidly so it is little surprise that they can do this with their eyes. Also, we have shown that unstructured environments (fields of moving dots) often yield results quite different than those of more structured ones (e.g., arrays of schematic trees; Cutting et al., 1997; Vishton \& Cutting, 1995; Li \& Warren, 2001). In particular, dot fields may introduce biases not present in more naturalistic stimuli. Given that responses are often different in the two situations, we would expect eye movements to differ as well.

What eye fixation data are relevant? The data of Wagner et al. (1981) derive from an ecological survey of 800 fixations by 16 people walking through a familiar setting (a town and campus) for $90 \mathrm{~min}$. These data suggest that $90 \%$ of the time we are looking away from our aim point by more than $5^{\circ}$. This is a conundrum, since the physical constraints of pedestrian locomotion suggest that we must know heading within $3.5^{\circ}$ (Cutting et al., 1992). If knowledge of heading is so important to us why do we spend so much of our time looking where it is not? The argument made here, and in our previous research, is that the best information for one's heading is indeed often well off one's path. Since the environment is typically a plenum of attention-grabbing objects, most of which are off one's path, one can well afford looking at them for their interest value while simultaneously gathering information about heading. Thus, we have focused on the observer-relative information off one's path.

\subsection{Invariants and Eye Movements}

Cutting et al (2000) investigated observers' eye movements during the course of simulated travel towards a grove of four trees and prior to making a heading judgment. Stimulus sequences mimicked pursuit eye fixations on one tree, which varied in screen position as shown in Figure 6a, during forward movement. This manipulation varied initial eye position within the stimulus array allowing for saccades elsewhere, and partly overcame the general tendency for observers to continue to look at midscreen. Important comparisons were between trees across trials in the same array position that were and were not part of invariant pairs. 

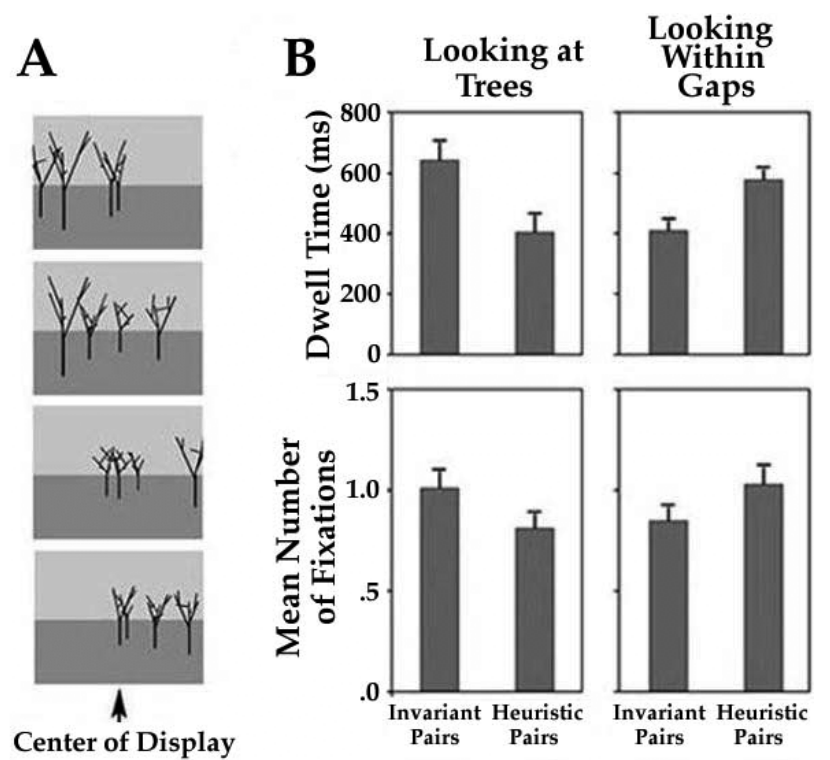

Figure 6. Panel A shows four sample arrangements of the four trees on given stimulus trials. Initial fixation was at screen center and would fall on one tree at the beginning of the trial. This variation allowed placement of invariant pairs on and off the initial fixation point. Panel B shows mean dwell times and mean numbers of fixations on each tree within invariant pairs and heuristic pairs, and within each gap between invariant pairs and heuristic pairs. Error bars indicate one standard error of the mean.

Wearing an eye-tracker, each of twelve participants looked anywhere on the screen they liked during the course of the trial; they determined their heading during the course of the trial, and at the end of the sequence moved the screen cursor to their apparent heading and pressed the mouse. Thus, two types of response were recorded - heading judgments at the end of each trial and eye fixations throughout each trial. Figure $6 \mathrm{~b}$ shows the results.

Observers spent most of their time (63\%) looking at one of the four trees, and considerably less time (24\%) looking in gaps between them. Given the resolution of the eye tracker the residual dwell time (13\%) was indeterminate. More importantly, as shown in Figure 6b, observers looked $240 \mathrm{~ms}$ longer at, and produced 0.18 more fixations on, each tree that was a member of an invariant pair than each heuristic-pair tree in its same stimulus position. From Figures $1 \mathrm{~b}$ and $1 \mathrm{c}$ such a result makes sense if observers are seeking reliable information about the location of their heading. In addition, mean dwell times were $170 \mathrm{~ms}$ shorter in gaps between invariant pairs and there was a mean of 0.17 fewer fixations there as well. This too makes sense. Figure $1 \mathrm{~b}$ shows that heading can never lie between members of invariant pairs, so there is little need to spend much time looking there. 
Finally, consider the relation between fixations and heading responses. For each observer, we tallied a 2X2 table: whether they looked at the near tree of the invariant pair or not, and whether they placed their heading response to the correct side of this tree or not. When observers looked at the pertinent tree they were $75 \%$ correct; when they did not look at this tree, they were only $19 \%$ correct. All observers showed this pattern. These patterns strongly suggest that observers seek out their heading through the use of invariant information among pairs of objects as they move through the environment.

\section{OVERVIEW}

Across the studies reported here on the information available in the retinal array during locomotion, we have shown that:

(a) the observer-relative motion of invariant generating pairs of stationary objects specifies nominal heading;

(b) the observer-relative motion of heuristic generating pairs offers probabilistic heading information;

- the invariants and heuristic can be used by observers in judgments about their heading;

- couplings of invariant pairs on either side of the heading with opposite sign constrain observer judgments to the narrow region between them, and these constraints are honored;

(c) a collection of many invariant pairs can, when yielding an incoherent heading solution due to the independent motion of one object, guide the detection of that moving object; and

(d) observers seek out invariant pairs through patterns of eye movements and fixations prior to their heading judgments. The rationale for this appears to be that, by fixating objects belonging to invariant pairs, convergent and decelerating divergent motion can be more easily registered, depth order noted, and confirmatory information about heading accrued. We assume making accurate heading judgments is a task that requires scrutiny; no pop out occurs, at least at pedestrian speeds.

In this chapter we have presented an overview of our research program on how observers determine their heading, or the instantaneous location in the visual field of the point towards which they are moving. Most approaches to this problem use the global information available across the entire visual field. Ours, in contrast, is a piecemeal approach which focuses on the information generally available at the fovea and which can accrue over a sequence of fixations. In terms of the information available to the moving observer, there 
is no difference between these two types of approaches. In terms of information use, however, we believe ours is superior in that it accounts for both correct performance and for errors that observers make in experiments and, by extrapolation, in the real world.

\section{REFERENCES}

Best, C.J., Crassini, B., \& Ross, R.H.. (2002). The roles of static depth information and objectimage relative motion in perception of heading. J. Exp. Psychol. Hum. Percept. Perform., $28,884-901$.

Brenner, E. (1991). Judging object motion during smooth pursuit eye-movements: The role of optic flow. Vision Res., 31, 1893-1902.

Calvert, E.S. (1954). Visual judgments in motion. J. Inst. Nav., 7 , 233-251 \& 398-402.

Chapman, P.R. \& Underwood, G. (1998). Visual search in dynamic scenes: Event type and the role of experience in viewing driving situations. In G. Underwood (ed.) Eye Guidance in Reading and Scene Perception . pp. 369-394. Amsterdam: Elsevier Science.

Cutting, J.E. (1986). Perception with an Eye for Motion. Cambridge, MA: MIT Press.

Cutting, J.E., Alliprandini, P.M.Z., \& Wang, R.F. (2000). Seeking one's heading through eye movements. Psychon. Bull. Rev., 7, 490-498.

Cutting, J.E. \& Readinger, W.O. (2002). Perceiving motion while moving, or how heading invariants make optical flow cohere. J. Exp. Psychol. Hum. Percept. Perform.,, 26, 731747.

Cutting, J.E., Springer, K., Braren, P.A., \& Johnson, S.H. (1992). Wayfinding on foot from information in retinal, not optical, flow. J. Exp. Psychol. Gen., 121, 41-72 \& 129.

Cutting J.E., \& Vishton P.M. (1995). Perceiving layout and knowing distances. in W. Epstein \& S. Rogers (Eds.). Perception of Space and Motion. pp. 69-117. San Diego, CA: Academic Press.

Cutting, J.E., Vishton, P.M. \& Braren, P.A. (1995). How we avoid collisions with stationary and with moving obstacles. Psychol. Rev., 102, 627-651.

Cutting, J.E., Vishton, P.M., Flückiger, M., Baumberger, M., \& Gerndt, J. (1997). Heading and path information from retinal flow in naturalistic environments. Percept. Psychophys., 59, 426-441.

Cutting, J.E. \& Wang, R.F. (2000). Heading judgments in minimal environments: The value of a heuristic when invariants are rare. Percept. Psychophys., 62, 1146-1159.

Cutting, J.E., Wang, R.F., Flückiger, M., \& Baumberger, M. (1999). Human heading judgments and object-based motion information. Vision Res., 39, 1079-1105.

Frey, B.F. \& Owen, D.H. (1999). The utility of motion parallax information for the perception and control of heading. J. Exp. Psychol. Hum. Percept. Perform., 25, 445-460.

Gibson, J.J. (1954). The visual perception of objective motion and subjective movement. Psychol. Rev., 61, 304-314. 
Gibson, J.J. (1966). The Senses Considered as Perceptual Systems. Boston: Houghton Mifflin.

Gilden, D.L. \& Proffitt, D.R. (1989). Understanding collision dynamics. J. Exp. Psychol. Hum. Percept. Perform., 15, 372-383.

Grigo, A. \& Lappe, M. (1999). Dynamical use of different sources of information in heading judgments from retinal flow. J. Opt. Soc. Am. A, 16, 2079-2091.

Grossberg, S., Mingolla, E., \& Pack, C. (1999). A neural model of motion processing and visual navigation by cortical area MST. Cereb. Cortex, 9, 878-895.

Hildreth, E.C. (1992). Recovering heading for visually-guided navigation. Vision Res., 32, 1177-1192.

Hollands, M.A. Marplehorvat, D.E., Henkes, S., \& Rowan, A.K. (1995). Human eye movements during visually guided stepping. J. Mot. Behav., 27, 155-163.

Hooge, I.T.C., Beintema, J.A., \& van den Berg, A.V. (1999). Visual search of heading direction. Exp. Brain Res., 129, 615-628.

Kellman, P.J. \& Kaiser, M.K. (1995). Extracting object motion during observer motion: Combining constraints from optic flow and binocular disparity. J. Opt. Soc. Am. A, 12, 623-625.

Kim, N.-G., Turvey, M.T., \& Growney, R. (1996) Wayfinding and the sampling of optical flow by eye movements. J. Exp. Psychol. Hum. Percept. Perform., 22, 1314-1319.

Koenderink, J. J. \& van Doorn, A.J. (1975). Invariant properties of the motion parallax field due to the movement of rigid bodies relative to an observer. Optica Acta, 22, 773-791.

Land, M. (1992). Predictable eye-head coordination during driving. Nature, 359, 318-320.

Land, M. (1999). Motion and vision: Why animals move their eyes. J. Comp. Physiol. A, 185, 341-352.

Land, M. \& Lee, D.N. (1994). Where do we look when we steer? Nature, 369, 742-744.

Lappe, M., Bremmer, F., \& van den Berg, A.V. (1999). Perception of self-motion from visual flow. Curr. Trends Cognit. Sci., 3, 329-336.

Li, L. \& Warren, W.H. (2001). Perception of heading during rotation: Sufficiency of dense motion parallax and reference objects. Vision Res., 40, 3873-3894.

Llewellyn, K.R. (1971). Visual guidance of locomotion. J. Exp. Psychol., 91, 245-261.

Morris, G.H. (1990). Hunter Seat Equitation (3 ${ }^{\text {rd }}$ ed.). New York: Doubleday.

Motorcycle Safety Foundation. (1992). Evaluating, Coaching, and Range Management Instructor's Guide. Irvine, CA: Author.

Motter, B. (1993). Focal attention produces spatially selective processing in visual area V1, V2, and V4 in the presence of competing stimuli. J. Neurophysiol., 7, 2239-2255.

Patla, A.E. \& Vickers, J.N. (1997). Where and when do we look as we approach and step over an obstacle in the travel path? NeuroReport, 8, 3661-3665.

Perrone, J. \& Stone, L. (1994). A model of self-motion estimation within primate visual cortex. Vision Res., 34, 1917-1938.

Perrone, J. \& Stone, L. (1998). Emulating the visual receptive-field properties of MST neurons with a template model of heading estimation. J. Neurosci., 18, 5958-5975. 
Readinger, W.O., Chatziastros, A., Cunningham, D.W., Bülthoff, H.H., \& Cutting, J.E. (2002). Systematic effects of gaze-eccentricity on steering. J. Exp. Psychol. Appl., in press.

Regan, D.M. \& Beverley, K.I. (1982). How do we avoid confounding the direction we are looking with the direction we are going? Science, 215, 194-196.

Rieger, J.H. \& Lawton, D.T. (1985). Processing differential image motion. J. Opt. Soc. Am. A, $2,354-360$

Royden, C.S., Banks, M.S., \& Crowell, J.A. (1992). The perception of heading during eye movements. Nature, 360, 583-585.

Rushton, S.K., Harris, J.M., Lloyd, M.R., \& Wann, J.P. (1998). Guidance of locomotion on foot uses perceived target location rather than optic flow. Curr. Biol., 8, 1191-1194.

Schivelbusch, W. (1988). The Railway Journey. Berkeley, CA: University of California Press.

Underwood, G \& Radach, R. (1988). Eye guidance and visual information processing: Reading: visual search, picture perception, and driving, in G. Underwood (ed.) Eye Guidance in Reading and Scene Perception. pp. 1-27. Amsterdam: Elsevier Science.

Vishton, P.M. \& Cutting, J.E. (1995). Wayfinding, displacements, and mental maps: Velocity fields are not typically used to determine one's aimpoint. J. Exp. Psychol. Hum. Percept. Perform., 21, 978-995.

Wagner, M., Baird, J.C., \& Barbaresi, W. (1981). The locus of environmental attention. J. Environ. Psychol., 1, 195-201.

Wang, R.F. \& Cutting, J.E. (1999a). A probabilistic model for recovering camera translation. Comput. Graph. Im. Process. 76, 205-212.

Wang, R.F. \& Cutting, J.E. (1999b). Where we go with a little good information. Psychol. Sci., $10,72-76$.

Warren, W.H. (1998). The state of flow, in T. Watanabe (ed.) High-level Motion Processing. pp. 315-358. Cambridge, MA: MIT Press.

Warren, W.H. \& Hannon, D.J. (1988). Direction of self-motion is perceived from optical flow. Nature, 336, 162-163.

Warren, W.H., Kay, B.A., Zosh, W.D., Duchon, A.P., Sahuc, S. (2001). Optic flow is used to control human walking. Nat. Neurosci., 4, 201-202.

Warren, W.H. \& Saunders, J.A. (1995). Perceived heading in the presence of moving objects. Percept., 24, 315-331.

Wertheim, A. (1995). Motion perception during self-motion: The direct versus inferential controversy. Behav. Brain Sci., 17, 293-355. 